ISSN 0103-8478

\title{
Compressibilidade e elasticidade de um Vertissolo afetado pela intensidade de pastejo bovino
}

\author{
Compressibility and elasticity of a Vertissol affected by the intensity of grazing cattle
}

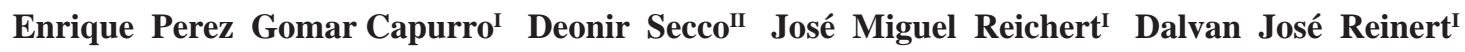

RESUMO

$O$ fornecimento de pastagem em quantidade $e$ qualidade em áreas de campo nativo é objetivo dos agropecuaristas. Neste trabalho, avaliou-se o impacto de três intensidades de pastejo por bovinos de corte, em área de campo natural melhorado com trevo branco e trevo vermelho, na densidade do solo (Ds), pressão de pré-consolidação ( $\sigma p)$, indice de compressão (Ic) e no indice de relaxação (Ir) de um Vertissolo derivado de basalto. A área está localizada no campo experimental pertencente ao INIA Glencoe, na região Centro-Norte do Uruguai (Latitude: $32^{\circ} 41$ 'S e Longitude: $\left.56^{\circ} 16^{\prime} \mathrm{O}\right)$. O solo tem profundidade de 0,92m, textura argilo-siltoso $\left(0,58 \mathrm{~kg} \mathrm{~kg}^{-1}\right.$ de argila) e estrutura granular. A intensidade de pastoreio configurou os tratamentos: sem pastoreio $(S P)$, pastoreio com baixa carga (que consiste no pastoreio de 20 animais por hectare de $385 \mathrm{~kg}$ aproximadamente de peso vivo, durante 4 dias) (PCB) e pastoreio com carga alta (40 animais por hectare de $385 \mathrm{~kg}$ aproximadamente de peso vivo durante 4 dias) (PCA). A análise estatística constou de comparações entre médias de Ds, $p$, Ic e Ir do solo nos três tratamentos, pelo teste t de Student a 5\% de significância. O PCA não provocou aumento de $D$ s em relação ao $S P$, nem alterações significativas na $\sigma p$ e no Ic, indicando que o maior volume do material orgânico na superficie do Vertissolo proporcionou alta capacidade de suporte da carga, baixa susceptibilidade à compactação e alta elasticidade do solo. O maior Ir da camada superficial em relação à subsuperficial condicionou maior capacidade de recuperação de deformação, pois o PCA não provocou alterações significativas na $\sigma p$ e no Ic, em relação ao $S P$ e $P C B$.

Palavras-chave: pressão de pré-consolidação, índice de compressão, indice de relaxação, campo nativo.

\section{ABSTRACT}

The supply of pasture quantity and quality in areas of native grassland is goal of ranchers. In this study we evaluated the impact of three grazing intensities by beef cattle in an area of unspoilt countryside with improved white clover and red cloveron soil bulk density ( $B d)$, pre-consolidation pressure (sp), compression index (Ci) and the relaxation index (Ri) in a Vertisol derived from basalt. The experiment was conducted in an area of unspoilt countryside with improved white clover and red clover, subjected to three intensities of grazing, located in the experimental field of INIA Glencoe, in the Centre-North of Uruguay (Latitude: $32^{\circ} 41^{\prime} S$ and Longitude: $56^{\circ} 16^{\prime} \mathrm{W}$ ). The soil has a depth of $0.92 \mathrm{~m}$, clayey silt $\left(0.58 \mathrm{~kg} \mathrm{~kg}^{-1}\right.$ clay) and has granular structure. The intensity of grazing configured the treatments: no grazing (SP), grazing with low load (consisting of grazing animals per hectare from 20 to approximately $385 \mathrm{~kg}$ of body weight, during 4 days) (PCB) and grazing with high load (40 animals per hectare of approximately $385 \mathrm{~kg}$ live weight, during 4 days) (PCA). Statistical analysis consisted of comparing averages of $B d, s p, C i$ and Ri in the three soil treatments by Student's t test at 5\% of significance. PCA caused no increase in relation to the Ds SP nor significant changes $\sigma p$ and Ic, indicating that the bulk of the organic material provided on the surface of Vertisoil high load bearing capacity, low compaction susceptibility and high elasticity of the soil. The most superficial layer of Ri with respect to subsurface conditioned more resilient deformation, because the PCA were no significant changes in $\sigma p$ and Ic against the SP and PCB.

Key words: preconsolidation pressure, compression index, index of relaxation, native grassland.

\section{INTRODUÇÃO}

O sistema de produção predominante na região Centro-Norte do Uruguai inclui o pastejo de bovinos e ovinos de forma extensiva em campo nativo. Os solos da região, de acordo com DURÁN (1985), variam em profundidade, encontrando-se Neossolos com profundidades inferiores a $25 \mathrm{~cm}$,

'Departamento de Solos, Centro de Ciências Rurais (CCR), Universidade Federal de Santa Maria (UFSM), Santa Maria, RS, Brasil.

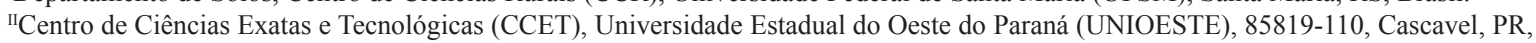
Brasil. E-mail: deonir.secco@unioeste.br. Autor para correspondência. 
até solos mais profundos, maiores que $45 \mathrm{~cm}$ de espessura, os Vertissolos.

Os Vertissolos desenvolvidos sobre basalto, no Uruguai, são solos profundos e caracterizam-se por apresentar altas porcentagens de argila expansiva em todo perfil (superiores a 50\%) e altos níveis de matéria orgânica, conferindo uma fertilidade natural elevada e uma estrutura granular forte, plásticos e muito suscetíveis à compactação em condições de alto conteúdo de água no solo (DURÁN, 1985; PUENTES et al., 1988). Entretanto, BRAIDA et al. (2008) afirmam que o maior teor de matéria orgânica aumenta a elasticidade do solo e, em consequência, reduz a susceptibilidade à compactação.

A susceptibilidade à compactação depende do tipo de solo, do conteúdo de água presente e do estado inicial de compactação (SECCO et al., 2009). Em condições de altos conteúdos de água no solo, a compactação provocada pelo pisoteio animal altera as propriedades físicas pelo efeito, repetitivo e cumulativo, do pisoteio excessivo sobre o solo. Em um Planossolo de várzea, o pisoteio animal ocasionou aumento da densidade e redução da porosidade total do solo, de acordo com VIZZOTTO et al. (2000), especialmente na camada de $0-5 \mathrm{~cm}$ de profundidade.

SILVA et al. (2000), em um Podzólico Vermelho-Amarelo, textura franco-arenosa, não observaram redução da produtividade do milho, devido à compactação imposta pelo pisoteio animal, embora tivesse ocorrido concentração de raízes na camada superficial da área sob sistema plantio direto. Porém, ALBUQUERQUE et al. (2001), em um Nitossolo Vermelho, sob integração lavoura-pecuária em área com sistema plantio direto, observaram que a compactação imposta pelo pisoteio animal reduziu o volume de macroporos e, em consequência, a condutividade hidráulica saturada com aumento da resistência do solo à penetração. Essas alterações físicas afetaram negativamente o crescimento e o rendimento de grãos do milho.

No entanto, as características acima mencionadas dos Vertissolos, como a expansão e contração, em função da variação do conteúdo de água, aliado ao alto teor de matéria orgânica presente, conferem grande resistência aos efeitos da compactação causada pelo pisoteio animal. Em termos de sistemas de produção pecuária extensiva, em que a base de produção de forragem é o campo nativo, ocorre acumulação significativa de material orgânico sobre a superfície, o que determina uma maior capacidade de suporte de carga sem promover danos irreversíveis à estrutura do solo pelo pisoteio animal.

A matéria orgânica do solo confere maior elasticidade e estabilidade dos agregados (SOANE, 1990). O campo nativo apresenta condições similares ao sistema plantio direto quanto ao acúmulo de material orgânico na superfície e baixa perda de solo por erosão (BAYER et al., 2000), além de conferir maior resistência do solo à compactação (BRAIDA et al., 2008, 2011).

Este trabalho teve por objetivo avaliar o impacto de três intensidades de pastejo por bovinos de corte, em área de campo natural melhorado, nos parâmetros de compressibilidade e elasticidade de um Vertissolo de basalto do Uruguai.

\section{MATERIAL E MÉTODOS}

Área de estudo e tratamentos

O experimento foi instalado em área de campo natural melhorado com as espécies Trifolium repens(trevo branco) e Trifolium pratense (trevo vermelho), implantadas no ano 2000, submetidas a três intensidades de pastoreio, localizada no campo experimental pertencente ao INIA Glencoe, Uruguai (Latitude: $32^{\circ} 41^{\prime} \mathrm{S}$ e Longitude: $56^{\circ} 16^{\prime} \mathrm{O}$ ). O solo é um Vertissolo de Basalto, com profundidade de $0,92 \mathrm{~m}$, com horizonte A de $0,19 \mathrm{~m}$ de espessura, textura argilo-siltoso e estrutura granular (Tabela 1).

A intensidade de pastoreio configurou os tratamentos SP: sem pastoreio, PCB: pastoreio com carga baixa (que consiste no pastoreio de 20 animais por hectare de $385 \mathrm{~kg}$ aproximadamente de peso vivo, durante 4 dias) e PCA: pastoreio com carga alta (40 animais por hectare de $385 \mathrm{~kg}$ aproximadamente de peso

Tabela 1 - Caracterização física e química do Vertisolo utilizado no experimento.

\begin{tabular}{|c|c|c|c|c|c|c|c|c|c|}
\hline \multirow{2}{*}{ Prof. (cm) } & \multicolumn{3}{|c|}{--------Granulometria $\left(\mathrm{g} \mathrm{kg}^{-1}\right)$------- } & \multirow{2}{*}{ PH Água } & \multicolumn{4}{|c|}{---Matéria Orgânica $\left(\mathrm{g} \mathrm{kg}^{-1}\right)$------- } & \multirow{2}{*}{$\mathrm{CaCO}_{3}(\%)$} \\
\hline & Areia & Silte & Argila & & Húmus & $\mathrm{C}$ & $\mathrm{N}$ & $\mathrm{C} / \mathrm{N}$ & \\
\hline $0-19$ & 131 & 463 & 406 & 6,2 & 6,89 & 39,9 & 2,9 & 13,8 & - \\
\hline $19-51$ & 74 & 274 & 652 & 6,8 & 3,12 & 18,1 & 1,3 & 13,9 & - \\
\hline $51-78$ & 71 & 284 & 645 & 7,5 & 1,74 & 10,1 & 0,9 & 11,2 & 0,29 \\
\hline 78-92 & 108 & 281 & 611 & 7,8 & 1,01 & 5,9 & 0,5 & 11,8 & 0,13 \\
\hline
\end{tabular}


vivo durante 4 dias). Os animais pastorearam sob estes regimes de pastoreio durante o inverno e primavera de 2003, dois anos após a implantação das espécies.

Amostragem e saturação inicial das amostras

Para determinação dos parâmetros de compressibilidade, coletaram-se 24 amostras com estrutura preservada, das quais 12 amostras na camada de 0-3cm (4 na área SP, 4 na área de PCB e 4 na área de PCA) e outras 12 amostras na camada de $9-12 \mathrm{~cm}$ (4 na área SP, 4 na área de PCB e 4 na área de PCA), em anéis volumétricos com $6,5 \mathrm{~cm}$ diâmetro e $2,0 \mathrm{~cm}$ de altura. As amostras foram coletadas ao acaso em cada tratamento somente dentro do perímetro circular irrigado.

As amostras foram então conduzidas ao laboratório, devidamente preparadas, saturadas com água durante $72 \mathrm{~h}$ e submetidas à tensão de $33 \mathrm{kPa}$ em câmara de Richards antes de se proceder aos ensaios de compressão uniaxial. Essa tensão foi utilizada para caracterizar a condição de umidade nas amostras no momento dos ensaios.

Ensaio de compressão uniaxial e determinação da pressão de pré-consolidação e índice de compressão

$\mathrm{O}$ ensaio de compressão uniaxial foi realizado segundo a norma brasileira de adensamentoNBR 12007/90 para condições de solo não saturado (ABNT, 1990), em uma prensa de adensamento uniaxial modelo S450 Terraload, equipada com um defletômetro da marca Starrett, com resolução de $0,0025 \mathrm{~mm}$ para determinação das medidas de deformação nas amostras.
A deformação das amostras foi medida depois de aplicadas pressões estáticas e sucessivas de $12,5,25,50,100,200,400$ e $1600 \mathrm{kPa}$ durante 5 minutos. Segundo SILVA et al. (2000), mais de 99\% da deformação máxima é alcançada em até 5 minutos depois de aplicada cada pressão.

Com base no deslocamento vertical, calcularam-se o índice de vazios e a densidade da amostra, com os quais foram obtidas as curvas de compressão para determinar o índice de compressão (Ic) e a pressão de pré-consolidação $(\sigma p)$ de cada uma delas, conforme método de Casagrande (HOLTZ \& KOVAKS, 1981; REICHERT et al., 2010).

A elasticidade do solo foi estudada nas mesmas amostras e condições em que foi estudada a compressibilidade, pelo índice de relaxação (Ir). Para este estudo, as cargas na prensa uniaxial foram aplicadas em três etapas: carregamento até a carga de $400 \mathrm{kPa}$; descarregamento; carregamento até a carga de $800 \mathrm{kPa}$; descarregamento; e finalmente novo carregamento, incluindo a carga de $1600 \mathrm{kPa}$. A partir da curva de compressão, com indicações da reta virgem e da reta de descompressão (Figura 1), calculou-se o Ir, conforme BRAIDA et al. (2008). $\mathrm{O}$ índice de relaxação foi calculado pela equação: $I r=100 \frac{\Delta e d}{\Delta e C}$

Em que: $\mathrm{Ir}=$ índice de relaxação (\%); $\Delta$ ed $=$ variação do índice de vazios durante $o$ descarregamento;

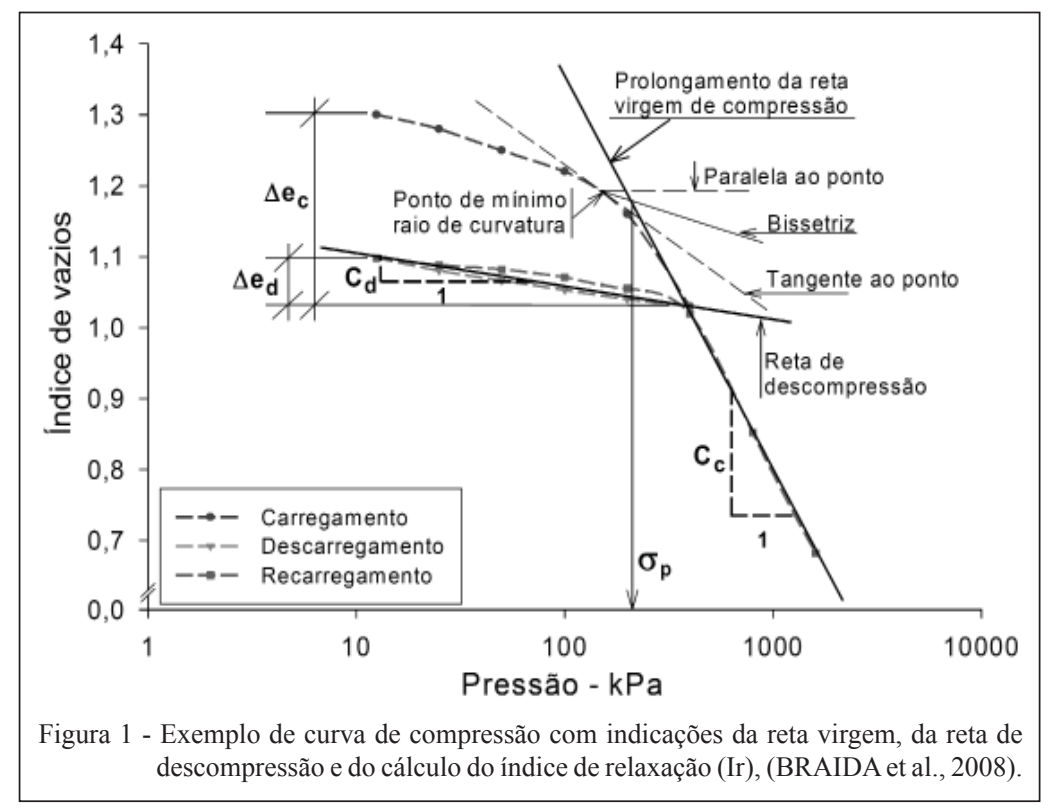

Ciência Rural, v.44, n.2, fev, 2014. 
$\Delta \mathrm{ed}=$ variação do índice de vazios durante $\mathrm{o}$ carregamento.

Análise dos dados

As análises estatísticas foram realizadas com auxílio do Software Sisvar, por meio de comparação de médias de tratamentos pelo teste $\mathrm{t}$ de student a 5\% de significância.

\section{RESULTADOS E DISCUSSÃO}

Os valores de densidade do solo, pressão de pré-consolidação, índice de compressão e índice de relaxação em duas profundidades e três intensidades de pastejo, equilibrados na tensão de $33 \mathrm{kPa}$ de umidade, constam na tabela 2.

\section{Densidade do solo (Ds)}

Os valores de Ds na camada de $0-3 \mathrm{~cm}$ não diferiram significativamente entre si nas três intensidades de pastejo avaliadas; na camada de 9-12 cm, o tratamento PCA apresentou valor superior ao SP e semelhante ao PCB (Tabela 2). Na camada de $0-3 \mathrm{~cm}$, a não ocorrência de diferença significativa pode estar associada ao maior volume de material orgânico e de raízes, os quais não permitiram alterações significativas nos valores de Ds. GOMAR et al. (2002), em área de campo natural melhorado no norte do Uruguai, sobre um Argissolo VermelhoAmarelo, verificaram aumento da Ds e redução na estabilidade do agregado com a redução do teor de matéria orgânica.
Na camada de 9-12cm, o maior valor de Ds no tratamento PCA em relação ao SP pode estar associado ao acúmulo das pressões impostas pelos animais, devido à maior intensidade de pastejo. Esses resultados corroboram os encontrados na literatura (VIZZOTO et al., 2000; SILVA et al., 2000; ALBUQUERQUE et al., 2001).

Os valores de Ds entre as camadas de $0-3 \mathrm{~cm}$ e $9-12 \mathrm{~cm}$, nos três tratamentos avaliados, foram diferentes significativamente, pois a camada de 9-12cm apresentou valores de Ds superiores à camada de $0-3 \mathrm{~cm}$, porém dentro da faixa de valores de densidade do solo $\left(1,25\right.$ a $\left.1,3 \mathrm{Mg} \mathrm{m}^{-3}\right)$, que apresenta indícios de que podem ocorrer restrições ao crescimento radicular em solos de textura argilosa, de acordo com REICHERT et al.(2009). Uma possível explicação se deva ao histórico das pressões impostas na área, onde a camada de $9-12 \mathrm{~cm}$ concentrou maiores deformações que a camada de $0-3 \mathrm{~cm}$. Outra possível explicação está no fato de que a camada de $0-3 \mathrm{~cm}$, por concentrar maior quantidade de material orgânico e raízes, condiciona menores valores de Ds em relação às camadas mais inferiores.

Pressão de pré-consolidação ( $\sigma p)$

Os valores de $\sigma p$ nas camadas de $0-3 \mathrm{~cm}$ e $9-12 \mathrm{~cm}$ não apresentaram diferenças significativas entre os tratamentos avaliados (Tabela 2).

A $\sigma p$ é também conhecida como capacidade suporte de carga dos solos. Nesse sentido, embora o tratamento PCA em relação ao SP tenha sido submetido a um maior pisoteio animal,

Tabela 2 - Densidade do solo (Ds, $\mathrm{Mg} \mathrm{m}^{-3}$ ), pressão de pré-consolidação ( $\sigma \mathrm{p}, \mathrm{kPa}$ ), índice de compressão (Ic) e índice de relaxação (Ir) em duas profundidades e três intensidades de pastejo, equilibrados na tensão de $33 \mathrm{kPa}$ de umidade (valores médios de 4 repetições).

\begin{tabular}{|c|c|c|c|c|c|}
\hline \multirow{2}{*}{ Variável } & \multirow{2}{*}{ Profundidade } & \multirow[b]{2}{*}{ SP } & \multirow[b]{2}{*}{ PCB } & \multirow[b]{2}{*}{ PCA } & \multirow[b]{2}{*}{ DMS } \\
\hline & & & & & \\
\hline \multirow[t]{3}{*}{ Ds } & $0-3$ & $0,91 \mathrm{Ab}$ & $0,92 \mathrm{Ab}$ & $0,94 \mathrm{Ab}$ & 0,10 \\
\hline & $9-12$ & $1,13 \mathrm{Ba}$ & $1,14 \mathrm{ABa}$ & $1,26 \mathrm{Aa}$ & 0,12 \\
\hline & DMS & 0,13 & & & \\
\hline \multirow[t]{3}{*}{$\sigma \mathrm{p}$} & $0-3$ & $35 \mathrm{Aa}$ & 39Aa & $44 \mathrm{Aa}$ & 10,37 \\
\hline & $9-12$ & 41Aa & $45 \mathrm{Aa}$ & 47Aa & 14,67 \\
\hline & DMS & 12,70 & & & \\
\hline \multirow[t]{3}{*}{ Ic } & $0-3$ & 0,70Aa & $0,62 \mathrm{Aa}$ & $0,61 \mathrm{Aa}$ & 0,16 \\
\hline & $9-12$ & $0,42 \mathrm{Ab}$ & $0,43 \mathrm{Aa}$ & $0,31 \mathrm{Ab}$ & 0,23 \\
\hline & DMS & 0,20 & & & \\
\hline \multirow[t]{3}{*}{ Ir } & $0-3$ & $0,21 \mathrm{Aa}$ & $0,22 \mathrm{Aa}$ & $0,22 \mathrm{Aa}$ & 0,07 \\
\hline & $9-12$ & $0,10 \mathrm{Ab}$ & $0,09 \mathrm{Ab}$ & $0,07 \mathrm{Ab}$ & 0,11 \\
\hline & DMS & 0,09 & & & \\
\hline
\end{tabular}

Médias de tratamentos seguidas de letras distintas, maiúsculas na linha e minúsculas na coluna, diferem pelo teste t-Student a $5 \%$ de significância. SP: sem pastoreio; PCB: pastoreio com carga baixa; PCA: pastoreio com carga alta; DMS: diferença mínima significativa. 
especialmente na camada de $0-3 \mathrm{~cm}$, não sofreu maiores deformações, conforme se verificou pelos valores de Ds, em consequência, apresentou valores de $\sigma p$ semelhantes. A possível explicação para a camada de 9-12 cm não apresentar diferença significativa entre os valores de $\sigma \mathrm{p}$, nos três tratamentos avaliados pode estar associada ao fato de o pisoteio animal não ter provocado alteração na estrutura do solo daquela camada, devido à baixa pressão imposta.

Embora os valores de $\sigma p$ da camada 9-12cm serem matematicamente superiores em relação à camada $0-3 \mathrm{~cm}$, nos três tratamentos avaliados, não foram diferentes significativamente. Valores superiores de $\sigma p$ na camada subsuperficial estão associados aos maiores valores de Ds dessa camada (Tabela 2), como corroboram os resultados encontrados na literatura que, à medida que ocorreu incremento da densidade do solo devido à compactação, ocorreu aumento nos valores da pressão de pré-consolidação (REICHERT et al., 2007; OLIVEIRA et al., 2011; PACHECO \& CANTALICE, 2011).

Índice de compressão do solo (Ic)

Em concordância com os valores de $\sigma p$ observados na camada de $0-3 \mathrm{~cm}$, verifica-se que os valores do Ic foram maiores no tratamento SP em relação aos demais. Nesse sentido, o Ic, que estima a suscetibilidade a compactação do solo, mostra maiores valores no tratamento SP, indicando que é mais susceptível a registrar maiores deformações que um solo mais compactado quando são submetidos à mesma pressão.

Quando são comparados os valores de Ic nas duas camadas, estes aparecem com maior valor na camada superficial $(0-3 \mathrm{~cm})$ em relação à camada subsuperficial $(9-12 \mathrm{~cm})$. De acordo com BRAIDA et al. (2008), os maiores conteúdos de carbono orgânico e biomassa radicular correspondentes ao estrato superior do solo podem conferir maior capacidade de deformação, assim como também uma maior elasticidade.

Índice de relaxação do solo (Ir)

Os valores do Ir foram estatisticamente semelhantes nas três intensidades de pastejo avaliadas e nas duas camadas (Tabela 2). Houve maior valor de Ir na camada de $0-3 \mathrm{~cm}$ em relação à camada de $9-12 \mathrm{~cm}$. Isso significa que o grande volume da material orgânico do solo propiciou maior capacidade de suporte de carga, sem formação de compactação adicional, pela maior elasticidade apresentada e, em consequência, pela maior facilidade de retorno à condição original após aplicação de uma pressão deformante.

$\mathrm{O}$ Ir refere-se à elasticidade do solo e sua capacidade de recuperar seu estado prévio à compactação e está associado ao maior conteúdo de carbono na superfície, como comentado anteriormente. Esses resultados corroboram os encontrados por BRAIDA et al. (2008), que afirmam que a elasticidade do solo está associada ao teor de carbono orgânico.

\section{CONCLUSÃO}

O PCAnão provocou aumento de densidade em relação ao SP, nem alterações significativas na $\sigma p$ e no Ic, indicando que o maior volume do material orgânico na superfície do Vertissolo proporcionou alta capacidade de suporte da carga, baixa susceptibilidade à compactação e alta elasticidade do solo. $\mathrm{O}$ maior Ir da camada superficial em relação à subsuperficial condicionou maior capacidade de recuperação de deformação, pois o PCA não provocou alterações significativas na $\sigma p$ e no Ic em relação ao SP e PCB.

\section{REFERÊNCIAS}

ABNT. NBR 12007: ensaio de adensamento unidimensional. Rio de Janeiro, 1990. 13p. Disponivel em: <http://pt.scribd. com/doc/89558364/Nbr-12007-Adensamento-Unidimensional>. Acesso em 23 out. 2013.

ALBUQUERQUE, J.A. et al. Efeitos da integração lavourapecuária nas propriedades físicas do solo e características da cultura do milho. R Bras Ci Solo, v.25, p.717-723, 2001. Disponível em: $<$ http://www.redalyc.org/articulo.oa?id=180218337022>. Acesso em 23 out. 2013. doi: 180218337022.

BAYER, C. et al. Efeito de sistemas de preparo e de cultura na dinâmica da matéria orgânica e na mitigação das emissões de $\mathrm{CO}_{2}$. R Bras Ci Solo, v.24, p.599-607, 2000. Disponível em: <http:// www.redalyc.org/articulo.oa? $\mathrm{id}=180218342013>$. Acesso em 23 out. 2013. doi: 180218342013.

BRAIDA, J.A. et al. Elasticidade do solo em função da umidade e do teor de carbono orgânico. R Bras Ci Solo, v.32, p.477-485, 2008. Disponível em: <http://www.redalyc.org/ articulo.oa? $\mathrm{id}=180214228002>$. Acesso em 23 out. 2013. doi: 180214228002

BRAIDA, J.A. et al. Matéria orgânica e seu efeito na física do solo. In: FILHO, O.K.et al.(Org.).Tópicos em ciência do solo. Viçosa: Sociedade Brasileira de Ciência do Solo, 2011. V.VII, p.222-227. Disponivel em: <http://www.fisicadosolo.ccr.ufsm.quoos.com.br/ downloads/Disciplinas/FisicaSolo/Braida_J.A_TCS2011.pdf $>$. Acesso em 23 out. 2013.

DURÁN, A. Los suelos del Uruguay. Montevideo: Editorial Hemisfério Sur, 1985. 398p. Disponivel: $<$ http://books.google.com. br/books?id=hJ9gAAAAMAAJ\&q=inauthor:\%22Artigas + R. + D ur\%C3\%A1n $\% 22 \&$ dq=inauthor:\%22Artigas + R. + Dur $\% \mathrm{C} 3 \% \mathrm{~A} 1$ $\mathrm{n} \% 22 \& \mathrm{hl}=\mathrm{pt}-\mathrm{BR} \& \mathrm{sa}=\mathrm{X} \& \mathrm{ei}=\mathrm{Gw} 1 \mathrm{oUuOIJ}$ fen $4 \mathrm{APriIFg} \& \mathrm{ved}=0 \mathrm{C}-$ DEQ6AEwAA >. Acesso em 23 out. 2013.

GOMAR, E.P. et al. Atributos do solo e biomassa radicular após quatro anos de semeadura direta de forrageiras de estação fria 
em campo natural dessecado com herbicidas. R Bras Ci Solo, v.26, p.211-223, 2002. Disponível em: <http://www.redalyc.org/ articulo.oa? $\mathrm{id}=180217643023>$. Acesso em 23 out. 2013. doi: 180217643023

HOLTZ, R.D.; KOVACS, W.D.An introduction to geotechnical engineering. New Jersey: Prentice-Hall, 1981. 733p. Disponivel $\mathrm{em}:<\mathrm{http}: / /$ www.amazon.com/Introduction-GeotechnicalEngineering-Robert-Holtz/dp/0132496348>. Acesso em 23 out. 2013.

OLIVEIRA, V.S. et al. Compressibiliade de um Argissolo distrocoeso submetido a diferentes manejos. R Bras Eng Agríc Ambiental, v.15, p.435-442, 2011. Disponivel em: $<$ http://dx.doi. org/10.1590/S1415-43662011000500001>. Acesso em 23 out. 2013. doi: 10.1590/S1415-43662011000500001.

PACHECO, E.P.; CANTALICE, J.R.B. Análise de trilha no estudo dos efeitos de atributos físicos e matéria orgânica sobre a compressibilidade e resistência à penetração de um Argissolo cultivado com cana-de-açúcar. R Bras Ci Solo, v.35, p.417428, 2011. Disponível em: <http://www.redalyc.org/articulo. oa? id=180218547011 $>$. Acesso em 23 out. 2013. doi: 180218547011 .

PUENTES, R. et al. Management of Vertisols in temperate regions. In: WILDING L.P.; PUENTES, R. (Eds.). Vertisols: their distribution, properties, classification and management.Texas: Texas A\&M University, 1988. p.129-145. Disponivel em: <http://books.google. com.br/books/about/Vertisols.html?id=ZM8QOgAACAAJ\&redir esc $=y>$. Acesso em 23 out. 2013.

REICHERT, J.M. et al. Compactação do solo em sistemas agropecuários e florestais: identificação, efeitos, limites críticos e mitigação. Tópicos Ci Solo, v.5, p. 49-134, 2007. Disponivel em: <http://www.fisicadosolo.ccr.ufsm.quoos.com.br/downloads/ Producao_Artigos/2007 Topicos.pdf. A>. Acesso em 23 out. 2013.
REICHERT, J.M. et al. Reference bulk density and critical degreeof-compactness for no-till crop. production in subtropical highly weathered soils. Soil \& Till Research, v.102, p.242-254, 2009. Disponivel: $<$ http://www.sciencedirect.com/science/article/pii/ S0167198708001025>. Acesso em 23 out. 2013. doi: 10.1016/j. still.2008.07.002.

REICHERT, J.M. et al. Mecânica do solo. In: REICHERT, J.M.et al. (Org.). Física do solo. Viçosa: Sociedade Brasileira de Ciência do Solo, 2010, V.1, p.29-102. Disponivel em: <http://www.sbcs. org.br/publicacao/fisica-do-solo/>. Acesso em 23 out. 2013.

SILVA, V.R. et al. Densidade do solo, atributos químicos e sistema radicular do milho afetados pelo pastejo e manejo do solo. R Bras Ci Solo, v.24, p.191-199, 2000. Disponível em: <http://www. redalyc.org/articulo.oa?id=180218272022>. Acesso em 23 out. 2013. doi: 180218272022 .

SECCO, D. et al. Atributos físicos e rendimento de grãos de trigo, soja e milho em dois Latossolos compactados e escarificados. Ciênc Rural, v.39,p.58-64, 2009. Disponivel em: <http://dx.doi. org/10.1590/S0103-84782009000100010>. Acesso em 23 out. 2013. doi: 10.1590/S0103-84782009000100010.

SOANE, B.D. The role of organic mater in soil compactibility: a reviw of some practical aspects. Soil Till Research, v.16, p.179201, 1990. Disponivel em: <http://dx.doi.org/10.1016/01671987(90)90029-D>. Acesso em 23 out. 2013. doi: 10.1016/01671987(90)90029-D

VIZZOTTO, V.R. et al. Efeito do pisoteio bovino em algumas propriedades físicas do solo de várzea.Ciênc Rural, v.30, p.965969, 2000. Disponível em: <http://dx.doi.org/10.1590/S010384782000000600007>. Acesso em 23 out. 2013. doi: 10.1590/ S0103-84782000000600007. 